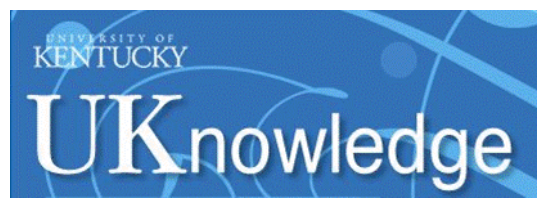

University of Kentucky

UKnowledge

6-1-1984

\title{
IUE Observations of DQ Herculis and its Nebula, and the Nature of the Cold Nova Shells
}

\author{
Gary J. Ferland \\ University of Kentucky, gary@uky.edu \\ R. E. Williams \\ University of Arizona \\ D. L. Lambert \\ University of Texas \\ G. A. Shields \\ University of Texas \\ M. Slovak \\ University of Texas
}

See next page for additional authors

Follow this and additional works at: https://uknowledge.uky.edu/physastron_facpub

Part of the Astrophysics and Astronomy Commons, and the Physics Commons

Right click to open a feedback form in a new tab to let us know how this document benefits you.

\section{Repository Citation}

Ferland, Gary J.; Williams, R. E.; Lambert, D. L.; Shields, G. A.; Slovak, M.; Gondhalekar, P. M.; and Truran, J. W., "IUE Observations of DQ Herculis and its Nebula, and the Nature of the Cold Nova Shells" (1984).

Physics and Astronomy Faculty Publications. 179.

https://uknowledge.uky.edu/physastron_facpub/179

This Article is brought to you for free and open access by the Physics and Astronomy at UKnowledge. It has been accepted for inclusion in Physics and Astronomy Faculty Publications by an authorized administrator of UKnowledge. For more information, please contact UKnowledge@lsv.uky.edu. 
IUE Observations of DQ Herculis and its Nebula, and the Nature of the Cold Nova Shells

Digital Object Identifier (DOI)
http://dx.doi.org/10.1086/162088

Notes/Citation Information

Published in The Astrophysical Journal, v. 281, no. 1, p. 194-204.

(c) 1984. The American Astronomical Society. All rights reserved.

The copyright holder has granted permission for posting the article here.

Authors

Gary J. Ferland, R. E. Williams, D. L. Lambert, G. A. Shields, M. Slovak, P. M. Gondhalekar, and J. W. Truran 
The Astrophysical Journal, 281 : 194-204, 1984 June 1

(C) 1984. The American Astronomical Society. All rights reserved. Printed in U.S.A.

\title{
IUE OBSERVATIONS OF DQ HERCULIS AND ITS NEBULA, AND THE NATURE OF THE COLD NOVA SHELLS
}

\author{
G. J. FerLand \\ Department of Physics and Astronomy, University of Kentucky \\ R. E. WiLliams \\ Steward Observatory, University of Arizona \\ D. L. Lambert, G. A. Shields, ${ }^{1}$ and M. Slovak \\ Department of Astronomy, University of Texas \\ P. M. GondhaleKar \\ Rutherford Appleton Laboratory \\ AND \\ J. W. TRURAN \\ Department of Astronomy, University of Illinois \\ Received 1983 August 26; accepted 1983 November 9
}

\begin{abstract}
The nebula ejected in the 1934 outburst of the classical nova DQ Her is remarkable for its unprecedentedly low temperature of $T_{e} 500 \mathrm{~K}$ as measured by Williams et al. In this paper, $I U E$ observations are combined with Steward optical spectra. We confirm that the gas is quite cold. We further show that the gas is ionized by the radiation field of the central object. We combine X-ray, ultraviolet, optical, and infrared observations of the underlying binary with the extreme-ultraviolet continuum deduced from the level of ionization of the nebula to obtain a composite energy distribution for the central object. This energy distribution bears no resemblance to that predicted by theoretical models of accretion disks. Photoionization models of the nebula using the deduced continuum, as well as theoretical accretion disk continua, are presented to show that the low electron temperature is the result of the very high metal abundances which characterize nova shells. Infrared fine-structure lines are efficient coolants, and low temperatures are achieved for a wide variety of radiation fields. The implications of these results for nebulae surrounding other old novae are discussed.
\end{abstract}

Subject headings: nebulae: individual - stars: individual — stars: novae - ultraviolet: spectra

\section{INTRODUCTION}

The nebula surrounding the 1934 nova DQ Herculis is the prototype of a class of nebulae whose spectra are characterized by the absence of collisionally excited forbidden lines, the presence of strong heavy-element recombination lines, and a sharp emission Balmer jump indicating an unprecedentedly low temperature for ionized gas $\left(T_{e}<10^{3} \mathrm{~K}\right.$, see Williams et al. 1978 ; Gallagher et al. 1980; Williams 1982a). Is the ionization a relic of an earlier phase? Perhaps recombination cannot keep pace with the nebular expansion and cooling (Williams et al. 1978). Is the gas photoionized by the radiation field emitted by the central object (see Ferland and Truran 1981)? To answer these questions requires a knowledge of the far-ultraviolet and soft $\mathrm{X}$-ray radiation fields of cataclysmic variables.

Cataclysmic variables are predicted to be strong sources of relatively hard radiation (see Pringle 1977; Pringle and Savonije 1979; Kylafis and Lamb 1979). The Einstein Observatory did not detect the majority of the old novae with the expected soft X-ray luminosity (Becker and Marshall 1981; Ferland et al. 1982). A study of the physical conditions in nebulae surrounding old novae should provide information on the nature of the unobservable far-ultraviolet radiation field.

\section{THE SPECTRUM OF DQ HER AND ITS NEBULA}

This section begins by describing new ultraviolet and optical observations of the DQ Her nebula, and then outlines the corrections necessary to produce a composite emission line

\footnotetext{
${ }^{1}$ Alfred P. Sloan Foundation Fellow.
}

spectrum. Lastly, ultraviolet observations of the central object are presented and combined with published optical and infrared data to measure the energy distribution of the underlying accreting binary.

\section{a) Ultraviolet Observations}

Observations of DQ Her with the International Ultraviolet Explorer were directed from the European Tracking Station of Villafranca, Spain on 1980 June 5. Details of these observations are summarized in Table 1. Both long and short wave cameras were used to cover the spectrum over the interval 1250-3000 $\AA$. Both exposures were obtained through the large $\left(10^{\prime \prime} \times 20^{\prime \prime}\right)$ aperture and were centered on DQ Her itself.

Figure 1 shows portions of the Photowrite reproductions of these observations. A strong continuum with overlapping emission lines is clearly present in both exposures. Since the continuum source is unresolved on the sky and the prominent

TABLE 1

ULTRAVIOLET OBSERVATIONS

\begin{tabular}{cccc}
\hline & & $\begin{array}{c}\text { Exposure } \\
\text { Imin) }\end{array}$ & $\begin{array}{c}\text { JD } \\
2,444,000+\end{array}$ \\
\hline SWP $9201 \ldots \ldots \ldots \ldots$ & Low & 370 & 395.9 \\
LWR $7981 \ldots \ldots \ldots \ldots$ & Low & 405 & 398.9 \\
SWP $6358 \ldots \ldots \ldots \ldots$ & Low & 90 & 118.9 \\
SWP $7408 \ldots \ldots \ldots \ldots$ & Low & 180 & 222.5 \\
LWR $7500 \ldots \ldots \ldots \ldots$ & Low & 180 & 344.0 \\
\hline
\end{tabular}

${ }^{\text {a }}$ All observations through the $10^{\prime \prime} \times 20^{\prime \prime}$ aperture. 

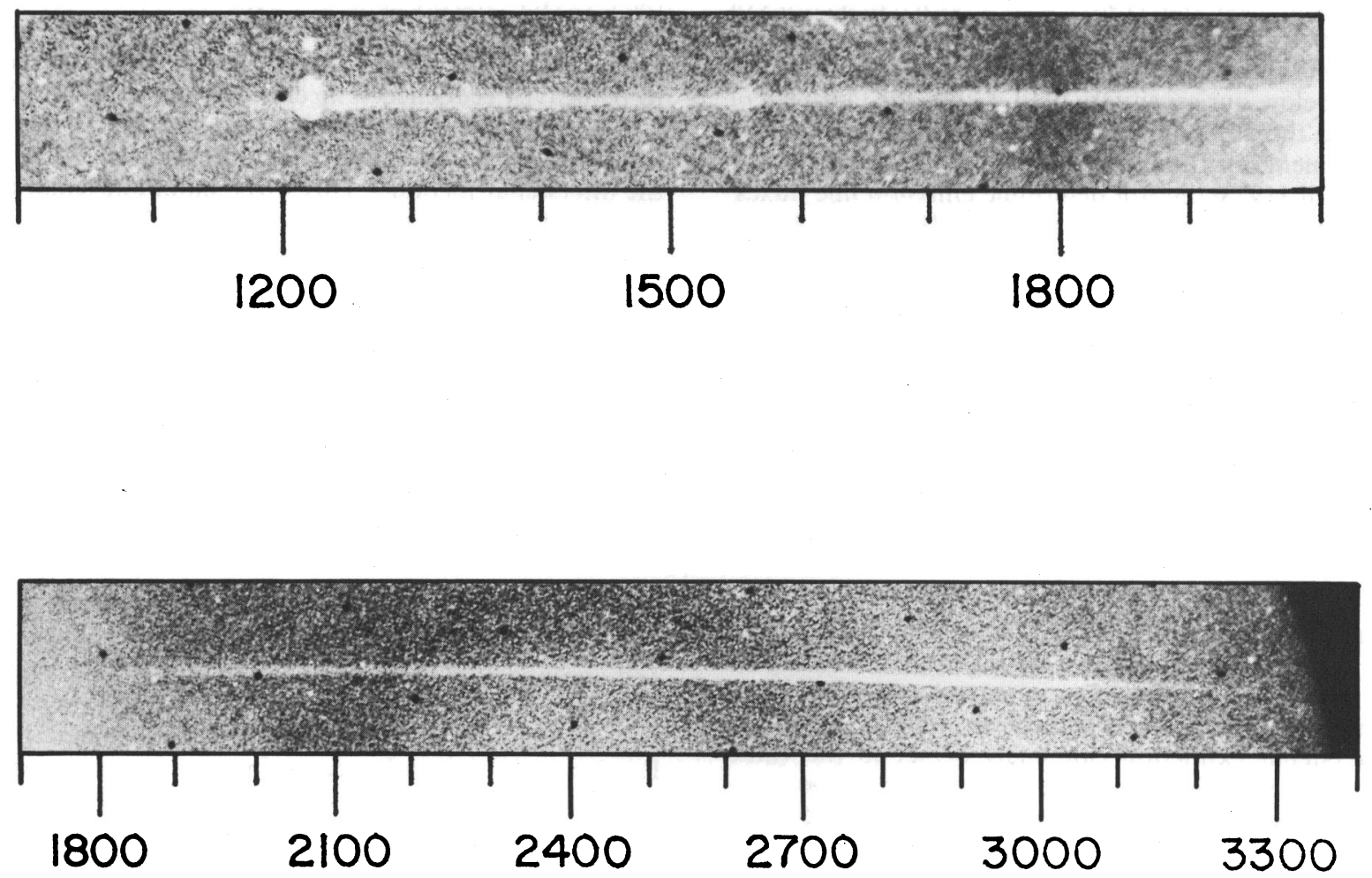

FIG. 1.-IUE images of ultraviolet spectra. This panel shows portions of IUE images SWP 9201 and LWR 7981 (upper and lower portions, respectively). The short-wave spectrum shows extended geocoronal Ly $1216 \AA$ and nebular emission at $1335 \AA$ which we identify as C II $1335 \AA$. This line is superposed on the unresolved line and continuum spectrum originating in the accretion disk. No extended emission is visible on the long-wave spectrum. 
emission-line spectrum is characteristic of an accretion disk, this spectrum is attributed to the binary system rather than the surrounding nebula.

Emission originating in the shell (size $20^{\prime \prime}$ ) will fill the entrance aperture and be spatially resolved on the twodimensional image. Extended geocoronal Ly $\alpha$ emission is present. The only feature clearly originating in the nebula is a weak line at $1335 \AA$ with an integrated flux of $1.1 \times 10^{-13} \mathrm{ergs}$ $\mathrm{cm}^{-2} \mathrm{~s}^{-1}$ and attributed to $\mathrm{C}$ II. We estimate the upper limits to the strengths of any indetected lines to be $0.3 \times 10^{-13}$ ergs $\mathrm{cm}^{-2} \mathrm{~s}^{-1}$ for both spectra.

Previous UV observations of the DQ Her nebula were made by Hartmann and Raymond (1981), who reported detections of both C II $1335 \AA$ and C III] $1909 \AA$. The flux they derived for the $C$ II line was roughly half of our value, and their strength for $\mathrm{C}$ III] was below our sensitivity limit. Given the faintness of the nebula and the different orientations of the entrance aperture, the agreement is satisfactory.

Williams et al. (1978) did not determine emission line fluxes, and therefore we reobserved the nebula. On 1982 May 23, we obtained spectra of the envelope using the Steward Observatory $2.3 \mathrm{~m}$ telescope with a Reticon array intensified by an RCA blue-sensitive image tube, at a resolution of $15 \AA$. The spectrometer slit $\left(20^{\prime \prime} \times 8^{\prime \prime}\right)$ was oriented east-west and placed in two positions, such that it was centered both $6^{\prime \prime}$ north and $6^{\prime \prime}$ south of the stellar system. The relative line strengths of the co-added spectra of the northern and southern segments of the shell are given in Table 2. The absolute $\mathrm{H} \beta$ flux of the combined portions of the shell we sampled was $6.2 \times 10^{-14}$ ergs $\mathrm{cm}^{-2} \mathrm{~s}^{-1}$.

\section{b) A Composite Emission-Line Spectrum}

To compare the strength of the $\mathrm{C}$ II line to those present in the optical spectrum, we must correct for both the effects of interstellar reddening and the different entrance aperture sizes. DQ Her lies at a high galactic latitude $\left(b=26^{\circ}, z=200 \mathrm{pc}\right)$ and the interstellar extinction can safely be set to the value derived for galaxies in that direction $\left(A_{v}=0.35 \mathrm{mag}\right.$, de Vaucouleurs, de Vaucouleurs, and Corwin 1976), and $A(1335 \AA)=$ $1.0 \mathrm{mag}$ (Seaton 1979). We estimate that the entrance aperture of the IUE spectrometer passed roughly half of the total flux from the nebula in both our observations and Hartmann and

TABLE 2

COMPOSITE SPECTRUM

\begin{tabular}{|c|c|c|}
\hline Ion & $\lambda(\AA)$ & Intensity $^{\mathrm{a}}$ \\
\hline $\mathrm{H}_{\mathrm{i}} \ldots \ldots \ldots \ldots \ldots \ldots$ & $\mathrm{Bac}$ & 155 \\
\hline $\mathrm{H}$ i $\ldots \ldots \ldots \ldots \ldots \ldots$ & 4340 & 65 \\
\hline $\mathrm{H}$ i $\ldots . .$. & 4861 & 100 \\
\hline $\mathrm{H}_{\mathrm{i}} \ldots \ldots \ldots \ldots \ldots \ldots$ & 6563 & 146 \\
\hline $\mathrm{He}_{\mathrm{i}} \ldots \ldots \ldots \ldots \ldots$ & 5876 & 26 \\
\hline He il $\ldots \ldots \ldots \ldots \ldots$ & 4686 & 18 \\
\hline $\mathrm{C}_{1} \ldots \ldots \ldots \ldots \ldots \ldots \ldots$ & 1656 & $<90$ \\
\hline $\mathrm{C}$ II $] \ldots \ldots \ldots \ldots \ldots$ & 2326 & $<90$ \\
\hline $\mathrm{C}_{\text {}} \mathrm{f} \ldots \ldots \ldots \ldots \ldots \ldots \ldots \ldots$ & 1335 & $270:$ \\
\hline $\mathrm{C}_{\mathrm{n}} \ldots \ldots \ldots \ldots \ldots \ldots$ & 4267 & 29 \\
\hline $\begin{array}{l}{ }_{n} \ldots \ldots \ldots \ldots \\
\ldots \ldots \ldots \ldots \ldots\end{array}$ & 1909 & $13::$ \\
\hline $\mathrm{N}_{\text {II }} \ldots \ldots \ldots \ldots \ldots \ldots \ldots$ & 5005 & 29 \\
\hline$[\mathrm{N}$ II $] \ldots \ldots \ldots \ldots \ldots$ & 6584,6548 & 38 \\
\hline$\left[\mathrm{O}_{1}\right] \ldots \ldots \ldots \ldots \ldots$ & 6300 & $<5$ \\
\hline$[\mathrm{O} \mathrm{II}] \ldots \ldots \ldots \ldots \ldots$ & 3727 & 100 \\
\hline
\end{tabular}

a Corrected for an assumed reddening of $A_{v}=$ 0.35 and expressed as intensities relative to $\mathrm{H} \beta=100$. We find $F(\mathrm{H} \beta)=6.2 \times 10^{-14} \mathrm{ergs} \mathrm{cm}^{-2}$ $\mathrm{s}^{-1}$.
Raymond's (1981) observations. We adopt a $\lambda 1335$ flux of $7.2 \times 10^{-14} \mathrm{ergs} \mathrm{s}^{-1} \mathrm{~cm}^{-2}$ as representative of half the shell. The corrections for the unobserved portions of the shell and interstellar extinction raise the total flux of the $\mathrm{C}$ II line to $\sim 3.6 \times 10^{-13} \mathrm{ergs}^{-2} \mathrm{sm}^{-1}$. We judge this flux to be uncertain by roughly a factor of 2 . The entrance aperture for the Steward observations sampled $60 \%$ of the nebula. The geometrical correction, together with the correction for interstellar reddening, brings the total $\mathrm{H} \beta$ flux up to $1.4 \times 10^{-13}$ ergs $\mathrm{cm}^{-2} \mathrm{~s}^{-1}$. We judge this flux to be uncertain by $\sim 30 \%$.

We know of no way to estimate the extinction due to internal dust in the nebula. This could be important since novae of the DQ Her type are generally believed to undergo an epoch of rapid grain formation (see Gallagher and Starrfield 1978), although the line of sight to the central object does not appear to be heavily reddened. The effects of any dust which survives today could be magnified by resonant scattering of $\lambda 1335$. Similarly, we do not correct for scattering of $\lambda 1335$ by $\mathrm{C}^{+}$in the interstellar medium. This effect should be small because of the large Doppler width of nova emission lines.

Table 2 lists the composite emission-line spectrum deduced here. The relative intensities of the optical lines should be accurate to $10 \%-15 \%$ because they do not depend on the absolute calibrations. The $\mathrm{C}$ II $1335 / \mathrm{H} \beta$ ratio could be in error by factors approaching $\sim 3$. The secular variability which characterizes this nebula has a time scale of 40 years and should not affect our analysis, but short-term cyclical variations (perhaps caused by changes in the underlying binary) could affect the optical/ultraviolet intensity ratios which are based on spectra taken 2 years apart.

\section{c) The Underlying Binary}

We observed the central object to determine the character and luminosity of the optical-UV radiation field. Details of the short and long wave exposures, all of which were obtained at Goddard Space Flight Center, are summarized in Table 1. Portions of the Villafranca exposures were overexposed and were not included in this part of the analysis. Continuum points free from emission lines were chosen, and averages made over $100 \AA$ intervals. These points were then corrected for the interstellar extinction of $A_{v}=0.35 \mathrm{mag}$ and plotted as in Figure 2. These observations represent averages over the rapid variations which characterize cataclysmic binaries. This figure also shows optical and infrared continuum points measured by Schneider and Greenstein (1979). The two groups of data agree well in overlapping regions, so we are confident that the figure provides the intrinsic ultraviolet-infrared continuum of this object. For comparison, a line indicating a power law with spectral index -2 is also drawn on the figure.

\section{PHYSICAL CONDITIONS IN THE NEBULA}

In this section several parameters characterizing the DQ Her nebula are deduced from the emission-line spectrum. Next the density and mass of the ionized gas are considered, and in the following subsection the carbon recombination spectrum is used to deduce a low temperature and fairly high level of ionization. In the last subsection we show that both the recombination and cooling time scales are shorter than the expansion time so that the nebula is likely to be in steady state. Lastly, we argue that the energy requirements to maintain a modest level of heating and ionization in the nebula are easily satisfied by the radiation field emitted by the underlying cataclysmic binary. 


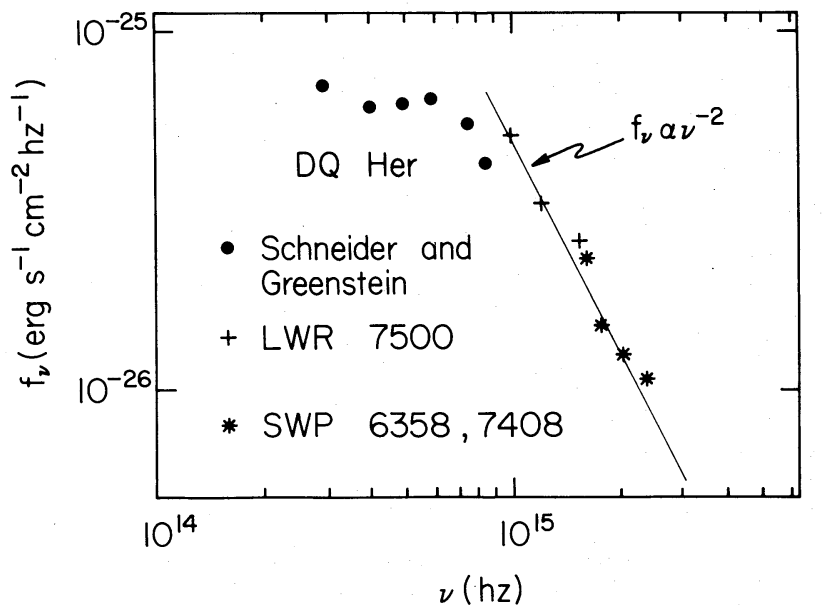

FIG. 2.-Composite ultraviolet-infrared continuum. This figure shows a composite ultraviolet, optical, and infrared continuum obtained by combining the published data of Schneider and Greenstein (1979) with new IUE observations. The points are reddening-corrected fluxes and are averages over emission-line free continuum intervals. For comparison, a line corresponding to a power law with spectral index -2 is drawn to fit the ultraviolet continuum.

\section{a) Basic Properties}

The envelope ejected in the 1934 outburst of DQ Her is now visible as an elliptical shell with radius $\sim 8^{\prime \prime}$ and a thickness $\delta r / r \approx 0.3$ (Williams et al. 1978). These numbers were measured halfway between the major and minor axis of the nebula and should be fairly representative. The nebular expansion parallax gives a distance of $d=420 \pm 100$ pc (Ferland 1980a), where the uncertainty is introduced by the elliptical shape of the nebula.

The volume of the shell and the $\mathrm{H} \beta$ luminosity can be used to estimate the gas density. The angular diameter and distance quoted above correspond to radii of

$$
\begin{aligned}
r_{\text {in }} & =3.7 \times 10^{16}(D / 420 \mathrm{pc}) \mathrm{cm}, \\
r_{\text {out }} & =5.1 \times 10^{16}(D / 420 \mathrm{pc}) \mathrm{cm},
\end{aligned}
$$

in turn corresponding to an effective emitting volume of

$$
V_{\text {eff }}=2.2 \times 10^{50}(\epsilon / 0.67)(D / 420 \mathrm{pc})^{3} \mathrm{~cm}^{-3} \text {. }
$$

In this expression $\epsilon=\frac{2}{3}$ is the volume filling factor estimated by examining the Steward photograph of the nebula. Combining the $\mathrm{H} \beta$ luminosity deduced above with the case $\mathrm{B} \mathrm{H} \beta$ emissivity for $T_{e}=500 \mathrm{~K}$ (Ferland $1980 \mathrm{~b}$ ), we find a density

$$
N_{p}=93\left(\frac{\epsilon}{0.67}\right)^{-1 / 2}\left(\frac{D}{420 \mathrm{pc}}\right)^{-1 / 2}\left(\frac{T}{500 \mathrm{~K}}\right)^{2 / 5} \mathrm{~cm}^{-3},
$$

where we have assumed $N_{e}=1.2 N_{p}$ and the temperature deduced by Williams et al. (1978).

The mass of ionized gas in the shell is

$$
M_{\text {shell }}=4.8 \times 10^{-5}\left(\frac{\epsilon}{0.67}\right)^{1 / 2}\left(\frac{D}{420 . \mathrm{pc}}\right)^{5 / 2}\left(\frac{T}{500 \mathrm{~K}}\right)^{2 / 5} M_{\odot},
$$

where we have assumed a mean mass per proton of $2 m_{p}$, which is appropriate for gas with the heavy-element enrichment typical of novae (Williams et al. 1978; Ferland and Shields 1978; Stickland et al. 1981). This mass is considerably higher than the mass of ionized gas deduced by Pottasch (1959, $\left.M=0.7 \times 10^{-5}, M_{\odot}\right)$ from his analysis of data obtained during much earlier phases of the outburst. This disagreement suggests that a good fraction of the gas in the nova envelope during early stages of the outburst was neutral, a view supported by the strength of such low ionization potential species as $\mathrm{Na} \mathrm{I}$ and $\mathrm{Ca}$ II in the original data (Payne-Gaposchkin and Whipple 1939).

\section{b) The Carbon Recombination Spectrum}

Williams et al. (1978) argued that the majority of the optical emission lines were formed by recombination at very low temperatures. This must also be the case for the ultraviolet spectrum since we see $2 p^{2} P^{o}-2 p^{2}{ }^{2} D \lambda 1335$ but not $2 p^{2} P^{o}-2 p^{2}{ }^{4} P$ 22324. Under conditions of collisional excitation at nebular temperatures $\left(T_{e}=10^{4} \mathrm{~K}\right)$ the unobserved intercombination line should have an intensity $\sim 100$ times larger than $\lambda 1335$ because of its lower excitation potential. Calculations to be described below show that $\lambda 2324$ is expected to be roughly 10 times weaker than $\lambda 1335$ (and hence undetectable on our spectra) when the lines are formed by recombination. A low temperature is further indicated by the weakness of C III] 1909 $\AA$ which is collisionally excited and much stronger than recombination $1335 \AA$ for classical nebular temperatures.

The most complete discussion of recombinations to $\mathrm{B}$ Isequence ions is that by Nussbaumer and Storey (1983), who include dielectronic recombination through the low-lying autoionizing levels which are accessible at nebular temperatures. Dielectronic recombination to $\mathrm{C}^{+}$is typically 4 times faster than radiative recombination at $T=10^{4} \mathrm{~K}$. The $\mathrm{C}$ II $1335 \AA$ line is strongly enhanced by this process, unlike the C II $4267 \AA$ line which is formed mainly by radiative recombination.

Over certain ranges of temperature the intensity ratio $I(1335) / I(4267)$ can be used to estimate $T_{e}$. For the temperature interval $7000<T_{e}<15,000 \mathrm{~K}$ (Storey 1981; see also Seaton 1978 ) the intensity ratio varies only between 56.3 and 59.8. The intensity ratio will fall dramatically when $T<10^{3} \mathrm{~K}$, because the autoionizing $3 d^{2} D^{o}$ and $3 d^{2} F^{o}$ states (which decay to produce the $1335 \AA$ line) lie $1770 \mathrm{~cm}^{-1}$ and $3300 \mathrm{~cm}^{-1}$ above the ionization limit, respectively, and are inaccessible to the free electrons. The fact that the observed ratio is $\sim 9( \pm 0.5 \mathrm{dex})$ rather than the $\sim 60$ predicted above suggests that the temperature is indeed low enough to suppress dielectronic recombination.

To estimate just how low the temperature must be to produce the observed ratio, we have computed $\mathrm{C}$ II recombination coefficients for the temperature range of interest. The $\lambda 4267$ coefficient was computed assuming simple hydrogenic radiative recombination using the program described by Ferland $(1980 b)$. These coefficients are shown in Figure 3 and are in excellent $(\sim 5 \%)$ agreement with those derived by Pengelly (as quoted by Seaton 1978).

Both radiative recombination and dielectronic recombination contribute to production of the $1335 \AA$ line. Radiative rates were again computed using the hydrogenic approximation. Dielectronic rates were computed assuming that the autoionizing levels are held in LTE with the continuum (see Storey 1981). In this calculation we have used energy levels listed in Moore (1970) and atomic transition probabilities computed by Kurucz and Peytremann (1975). These results are also shown in Figure 3 and are in good $(\sim 20 \%)$ agreement with those computed for the higher temperatures by Nussbaumer and Storey. The differences are attributable to the different sources of transition probabilities employed. For temperatures greater than $\sim 400 \mathrm{~K}, \lambda 1335$ is formed mainly by dielectronic recombination, and radiative recombination dominates at 


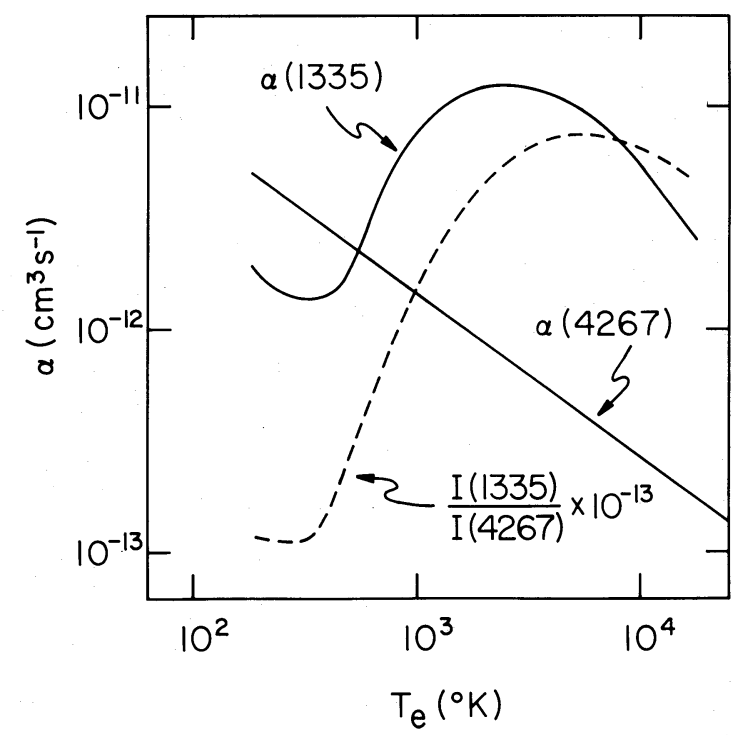

FIG. 3.-C II recombination spectrum. Recombination coefficients for the $\mathrm{C}$ in lines $4267 \AA$ and $1335 \AA$ are shown as a function of temperature. The 4267 line is formed mainly by radiative recombination over the entire range of temperature while the $1335 \AA$ line is formed largely by dielectronic recombination for $T>500 \mathrm{~K}$. The intensity ratio $I(1335 \AA) / I(4267 \AA)$ is also shown. The observed ratio is $9( \pm 0.5 \mathrm{dex})$ and corresponds to a temperature of $700 \mathrm{~K}$ $( \pm 0.3 \mathrm{dex})$. This temperature is consistent with that deduced by Williams et al. (1978) in their analysis of the optical spectrum.

lower temperatures. Figure 3 also shows the intensity ratio $I(1335) / I(4267)$. The observed intensity ratio is $9.3( \pm 0.5 \mathrm{dex})$ and corresponds to a temperature of $700 \mathrm{~K}( \pm 0.3 \mathrm{dex})$. This temperature is in good agreement with the Williams et al. (1978) estimate of $T_{e} \approx 500 \mathrm{~K}$, which we adopt.

We now estimate the level of ionization in the shell. In their discussion of a possible X-ray heated model for the DQ Her nebula, Ferland and Truran (1981) proposed that the shell was largely neutral and essentially all $\mathrm{C}^{++}$ions produced as the result of Auger ionization. As a test of their model they pointed out that the strongest ultraviolet line should be $\mathrm{C}_{\mathrm{I}}$ at $1656 \AA$, which we do not detect. The limit $I(1335) / I(1656)>3$ allows us to set a limit on the $\mathrm{C}^{++} / \mathrm{C}^{+}$ratio.

At low temperatures, the $\mathrm{C}$ I line will be formed mainly by radiative recombination; the lowest lying autoionizing level for $\mathrm{C}^{+}$is $2 p^{3}{ }^{1} D^{o}$, which lies some $7000 \mathrm{~cm}^{-1}$ above the ionization limit (Moore 1970), and is inaccessible at low temperatures. We estimate an effective radiative recombination coefficient of $9.0 \times 10^{-13} \mathrm{~cm}^{3} \mathrm{~s}^{-1}$ at a temperature of $500 \mathrm{~K}$ by scaling Aldrovandi and Péquignot's (1973) rates, using the arguments outlined by Williams $(1982 a)$. The ionic abundance ratio is

$$
\frac{N\left(\mathrm{C}^{++}\right)}{N\left(\mathrm{C}^{+}\right)}=\frac{I(1335)}{I(1656)} \frac{1335}{1656} \frac{\alpha^{\text {eff }}(1656)}{\alpha^{\text {eff }}(1335)}>1.3 .
$$

This shows that the shell must be fairly highly ionized.

Similarly, the C III] $1909, C_{\text {II }} 1335$ intensity ratio can be used to measure the $N\left(\mathrm{C}^{+3}\right) / N\left(\mathrm{C}^{+2}\right)$ ratio. The $\lambda 1909$ line will be formed mainly by radiative recombination at $T \approx 500 \mathrm{~K}$ since the radiative and dielectronic recombination rate coefficients are nearly equal at $1000 \mathrm{~K}$ (Aldrovandi and Péquignot 1973; Nussbaumer and Storey 1983). The effective recombination coefficient for $\lambda 1909$ will be roughly three-fourths the total since all triplet recombinations will eventually produce a $\lambda 1909$ photon. In this case $\alpha^{\text {eff }}(1909)=2.4 \times 10^{-11} \mathrm{~cm}^{3} \mathrm{~s}^{-1}$, and we find $N\left(\mathrm{C}^{+2}\right) / N\left(\mathrm{C}^{+3}\right)=45$ (i.e., essentially all the carbon is in the form of $\left.\mathrm{C}^{++}\right)$.

\section{c) Equilibrium Time Scales}

Williams et al. (1978) pointed out that in many respects the spectrum of the nebula resembles that of a nebula in a state of frozen-in ionization (i.e., the gas expands more rapidly than it recombines). Photoionization of the gas by the hard radiation produced in the accretion flow onto the white dwarf may also maintain the ionization (Ferland and Truran 1981). Here, we examine the relevant time scales for equilibrium, and the nature of the far-ultraviolet radiation field of this cataclysmic binary.

The nebula has shown $\mathrm{He}$ II $\lambda 4686$ emission for virtually its entire history (see the summary in Ferland and Truran 1981). The recombination time scale for the $\mathrm{He}^{++}$ion will be among the shortest and will provide the most severe demand on the presence or absence of an ionization source. Assuming $T_{e}=$ $500 \mathrm{~K}$ and the case A He II recombination coefficients listed by Ferland (1980b), we find

$$
\tau_{\mathrm{rec}} \approx\left(N_{e} \alpha_{A}\right)^{-1} \approx 22\left(\frac{N_{e}}{100 \mathrm{~cm}^{-3}}\right)^{-1}\left(\frac{T}{500 \mathrm{~K}}\right)^{-0.6} \mathrm{yr} .
$$

For comparison the expansion time for the nebula is $\sim 50$ years. The continued presence of $\lambda 4686$ suggests that the gas is being continuously ionized.

A stronger argument for photoionization is provided by the time scale required for the shell to radiate away its thermal energy. The internal energy of the nebula is

$$
E_{t} \approx 3 / 2 k T\left(M_{\text {shell }} / 2 m_{p}\right)=3 \times 10^{33} \text { ergs } .
$$

Since $\mathrm{H} \beta$ amounts to roughly $10 \%$ of the optical line emission from the shell, the rate at which energy is being radiated is

$$
L_{\text {shell }}>10 L(\mathrm{H} \beta) \approx 10 \times 4 \pi d^{2} F(\mathrm{H} \beta)=3 \times 10^{31} \mathrm{ergs} \mathrm{s}^{-1},
$$

and therefore the time scale for a significant temperature decline is

$$
\tau=\frac{E_{t}}{10 L(\mathrm{H} \beta)}=\frac{3 \times 10^{39} \mathrm{ergs}^{-1}}{3 \times 10^{31} \mathrm{ergs} \mathrm{s}^{-1}} \approx 3 \text { years } .
$$

This is actually a strong upper limit because we have not accounted for the luminous fine-structure lines which models predict should occur in the infrared. This time interval is substantially less than the lifetime of the shell, and clearly a heating source must exist for the gas.

\section{THE NATURE OF THE DQ HER SYNDROME}

Ferland and Truran (1981) proposed a model for the DQ Her nebula in which a very hard X-ray source ionized the shell but produced very little heating. In their model the putative $\mathrm{X}$-ray emission was produced by radial accretion onto the poles of a magnetic white dwarf, as outlined by Fabian, Pringle, and Rees (1976) and Kylafis and Lamb (1979). This picture was supported by the $71 \mathrm{~s}$ pulsation, which is commonly interpreted in terms of accretion onto an oblique rotator (Patterson, Robinson, and Nather 1978).

We can now reject the Ferland and Truran (1981) model on several grounds. As described in $\S$ III $b$, the ultraviolet carbon spectrum shows that the gas is actually fairly highly ionized $\left[N\left(\mathrm{C}^{++}\right) / N\left(\mathrm{C}^{+}\right)>1.5\right]$. The $\mathrm{X}$-ray model relied on Auger production of $\mathrm{C}^{++}$from neutral $\mathrm{C}$ and hence required that the gas be quite neutral. Furthermore, recent Einstein observations have established that DQ Her is not a detectable X-ray source (Córdova, Mason, and Nelson 1981); quiescent classical novae as a group do not radiate the soft X-ray luminosity predicted by simple accretion pictures (Ferland et al. 1982). Another diffi- 
culty with the Ferland and Truran model is that it is now clear that a low temperature characterizes nebulae surrounding many novae (Williams 1982a; see also Cohen and Rosenthal 1983); an explanation for DQ Her cannot therefore rely on features unique to DQ Her itself.

In this section, we show that cold ionized gas is a natural consequence of a very high metal abundance. Photoionization calculations show that, for a wide variety of ionizing radiation fields, the nebula will equilibriate at a temperature $T<10^{3} \mathrm{~K}$ when the heavy elements are strongly enriched and the density low enough for infrared fine-structure lines to cool the gas $\left(N_{e}<10^{4} \mathrm{~cm}^{-3}\right)$. The next two sections describe the ionizing continua used in our calculations, while $\S \S \operatorname{IV} c$ and IVd describe the calculations and results.

\section{a) The Ionizing Radiation Field in Theory}

The net radiation field from an accreting binary has been considered by, among others, Tylenda (1977), Kiplinger (1979), and Pringle and Savonije (1979) for classical accretion onto the equator of a white dwarf (applicable to the majority of old novae), and by Kylafis and Lamb (1979) for radial accretion (thought to apply to DQ Her itself). The radiation field results from the superposition of two components, a relatively soft (ultraviolet, optical, infrared) continuum produced by the accretion disk, and a much harder continuum ( $\sim$ soft X-ray) produced by the boundary layer or magnetic pole of the white dwarf.

In steady state the accretion disk continuum is set by the mass transfer rate, the white dwarf's mass, and, to a lesser extent, the gas viscosity. This follows because the disk radiates as a set of blackbodies, except perhaps for an optically thin outer boundary (Williams 1980). The boundary layer continuum, which is formed where the accreted gas comes to a rest on the surface of the white dwarf, is more difficult to predict since it depends on the area of the boundary layer, the mass flux in the region, any magnetic fields present, as well as the transfer of radiation through the accretion flow (Pringle 1981).

Figure 4 shows emergent continua calculated for the conventional case of mass transfer onto the equator of a nonmagnetized, nonrotating, solar mass white dwarf (Pringle 1977; Bath, Pringle, and Whelan 1980). This model is the simplest and is believed to describe most classical novae (Pringle 1981). It does not completely account for the optical and ultraviolet continua from several dwarf novae (Bath, Pringle, and Whelan 1980, Hassall et al. 1983). We shall see that it completely fails to account for the continuum observed from DQ Her.

The thresholds for photoionization of $\mathrm{H}^{0}, \mathrm{He}^{0}$, and $\mathrm{He}^{+}$, and the energy range of the IPC detector on board the Einstein Observatory, are shown in Figure 4. It is clear that all continua produce a significant flux of ionizing radiation, and even $\mathrm{He}^{+}$ can be ionized for $\dot{M}>10^{16} \mathrm{~g} \mathrm{~s}^{-1}$. This shows that the underlying binary can easily support the ionization of a significant shell (see also Tylenda 1977). For comparison with Figure 4, Smak (1982) and Patterson (1984) estimate $\dot{M}=10^{17.5} \mathrm{~g} \mathrm{~s}^{-1}$ for DQ Her, a rate characteristic of classical novae.

\section{b) The Radiation Field in Practice}

If the nebula is both in steady state and optically thin, then the level of ionization provides constraints on the ionizing radiation field. In $\S$ IIII $b$ we found that $N\left(\mathrm{C}^{++}\right) / N\left(\mathrm{C}^{+}\right)>1.3$. The photoionization-recombination balance equation between these ionization stages takes the familiar form

$$
\Gamma\left(\mathrm{C}^{+}\right) N\left(\mathrm{C}^{+}\right)=N_{e} \alpha N\left(\mathrm{C}^{++}\right),
$$

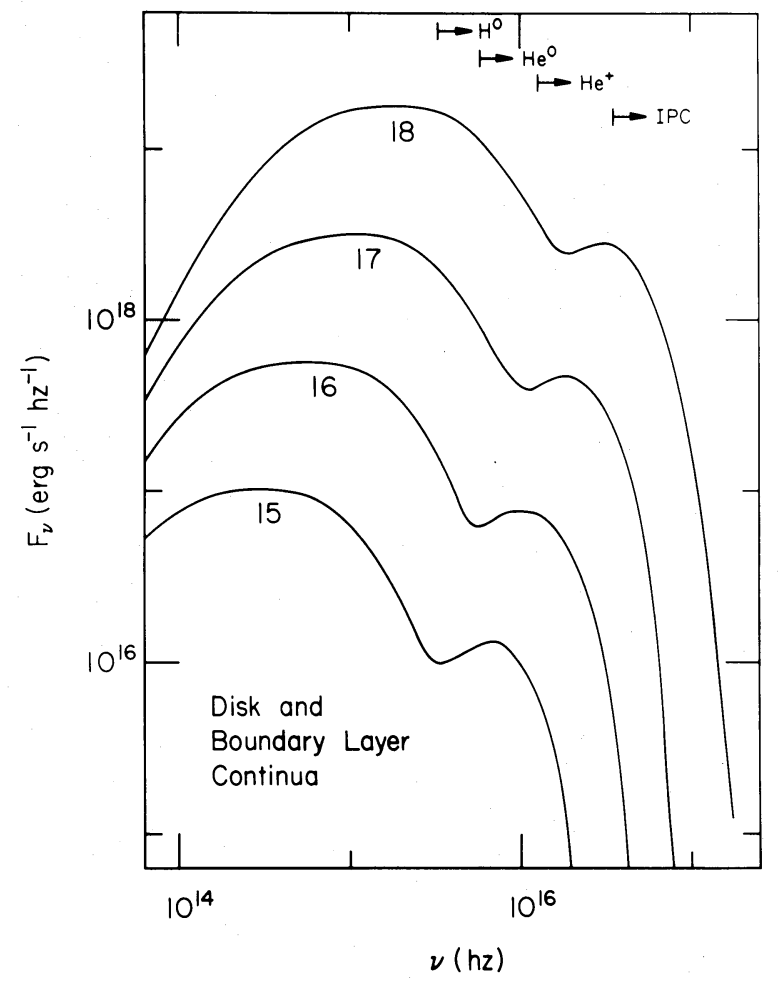

Fig. 4.-Accretion disk continua. This figure shows the emergent continuum, radiated into $4 \pi$ steradians, from a solar-mass white dwarf accreting matter in steady state. The white dwarf is assumed to be nonmagnetized and nonrotating. The log of the mass transfer rate $\left(\mathrm{g} \mathrm{s}^{-1}\right)$ is indicated next to each curve. The upper portion shows ionization edges of $\mathrm{H}^{0}, \mathrm{He}^{0}$, and $\mathrm{He}^{+}$, as well as the detector range of the IPC on board the Einstein Observatory.

where $\Gamma$ and $\alpha$ are the photoionization rate integral and recombination coefficient. If we approximate $\Gamma$ as

$$
\Gamma\left(\mathrm{C}^{+}\right)=\phi_{\text {ion }} \sigma \delta v=\phi_{\text {ion }} \sigma v
$$

where $\phi$ is the surface density of ionizing photons $\left(\mathrm{cm}^{-2} \mathrm{~s}^{-1}\right.$ $\mathrm{Hz}^{-1}$ ) and $\sigma$ is the photoionization cross section, then

$$
v F_{v}=4 \pi\langle r\rangle^{2} h v \frac{\Gamma}{\sigma}=4 \pi\langle r\rangle^{2} \frac{h v}{\sigma} N_{e} \alpha\left[\frac{N\left(\mathrm{C}^{++}\right)}{N\left(\mathrm{C}^{+}\right)}\right],
$$

where $\langle r\rangle \approx 4 \times 10^{16} \mathrm{~cm}$. For the parameters estimated here $\left[N_{e}=10^{2} \mathrm{~cm}^{-3}, \sigma=3 \times 10^{-18} \mathrm{~cm}^{-2}, \alpha(500 \mathrm{~K})=1.6 \times\right.$ $\left[N_{e}=10^{2} \mathrm{~cm}^{-3}, \sigma=3 \times 10^{-18} \mathrm{~cm}^{-2}, \alpha(500 \mathrm{~K})=1.6 \times\right.$ $h v=30 \mathrm{eV}$. Similarly the $1909 / 1335$ intensity ratio can be used to measure the continuum at $\sim 50 \mathrm{eV}$. In $\S$ III $b$ we found $N\left(\mathrm{C}^{+2}\right) / N\left(\mathrm{C}^{+3}\right)=45$, and argued that radiative recombination dominates in the formation of $\mathrm{C}^{+2}$. The arguments outlined above then lead to a luminosity of $1 \times 10^{32} \operatorname{ergs~s}^{-1}$ at $50 \mathrm{eV}$. Both extreme-ultraviolet fluxes are uncertain by $\sim 0.5$ dex.

These points are shown in Figure 5, along with the X-ray upper limit reported by Córdova, Mason, and Nelson (1981). In order to compare our observed ultraviolet-infrared continuum with these fluxes, a geometrical correction for the orientation of the optical continuum source must be made. If current ideas concerning DQ Her's geometry are correct, then the measures shown in Figure 2 reflect a nearly edge-on disk, while the continuum estimated from the nebula will represent a much more nearly face-on aspect. Estimates of the inclination of the DQ Her system tend to cluster around $i=80^{\circ}$ (Hutchings, Cowley, and Crampton 1979), and we have scaled the contin- 


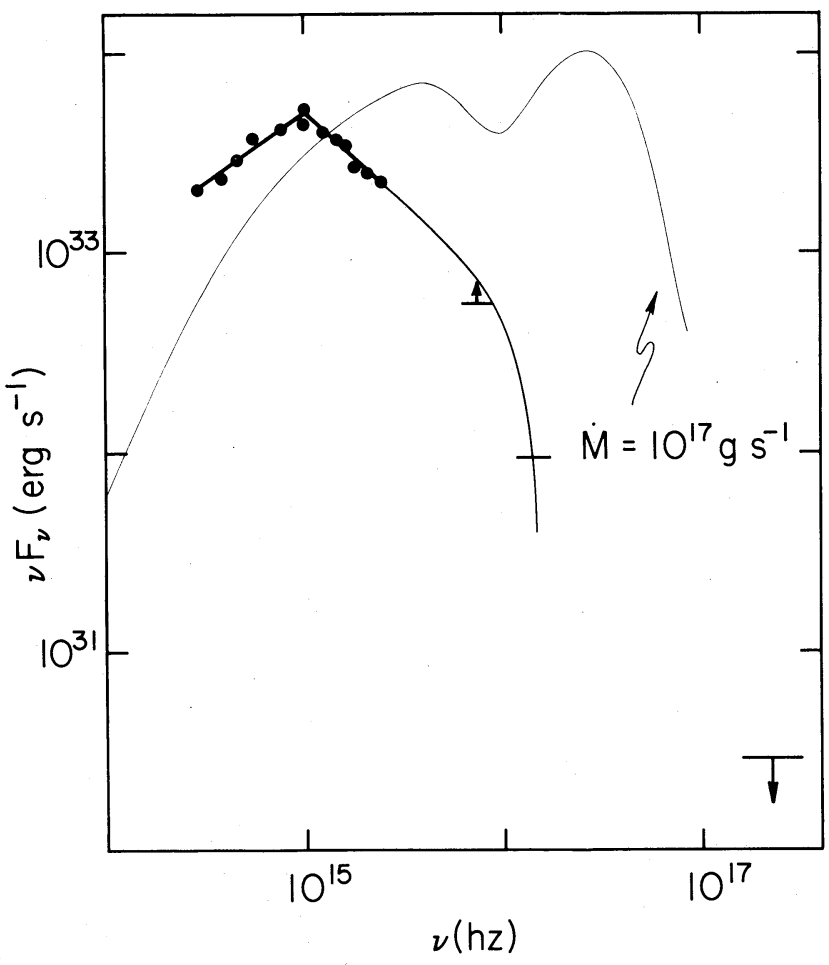

FIG. 5.--Observed continuum. The low-frequency $(14.4<\log v<15.4)$ points are the direct ground-based and $I U E$ observations described in the text These data have been corrected for an assumed reddening of $E(B-V)=0.12$ mag. A second correction for the assumed geometry (a flat disk seen at an inclination of $80^{\circ}$ ) has also been made. The X-ray upper limit is from Córdova et al. (1981), while the extreme-ultraviolet points are deduced from the level of ionization in the nebula. The ionizing continuum used in our calculations is also shown. Note that the vertical axis is $v F_{v}$ rather than $F_{v}$ as in Fig. 4. A theoretical accretion disk continuum corresponding to a mass transfer rate of $10^{17} \mathrm{~g} \mathrm{~s}^{-1}$ is also plotted to facilitate comparison between theory and observation.

uum in Figure 2 up by a factor of 5.8 to account for this. This of course assumes that the continuum source is a truly flat disk and that the form of the energy distribution is not affected by viewing angle effects.

It is apparent that the energy distribution in Figure 5 bears little resemblance to those predicted in the last section. Indeed, the theoretical infrared-uv continua are either flat or rising (asymptotically as $f_{v} \propto v^{+1 / 3}$ ) while we observe a continuum which is falling as a power law $\left(f_{v} \propto v^{-2}\right.$ in the ultraviolet). Note that, when the model continua do fall at higher frequencies, the flux follows an exponential (the Wein tail of the Planck function) rather than a power law. We have plotted a theoretical accretion disk continuum corresponding to a mass transfer rate of $10^{17} \mathrm{~g} \mathrm{~s}^{-1}$ on Figure 5 to underscore this difference. (Fabbiano et al. 1981 and Bianchini and Sabbadin 1982 find quite similar energy distributions in AM Her and GK Per, respectively.) The origin of this discrepancy is beyond the scope of this paper. Fortunately, models of the nebula are insensitive to the precise form of the ionizing continuum.

First, we shall consider one implication of Figure 5. The bolometric luminosity of DQ Her is $L_{\text {bol }} \approx 1 \times 10^{34} \mathrm{ergs} \mathrm{s}^{-1}$ by direct measurement. If DQ Her is indeed powered by accretion, then $L_{\text {bol }}=G M \dot{m} / r$, where $M, r$, and $\dot{m}$ are the white dwarf mass, white dwarf radius, and mass transfer rate, respectively. This result is independent of details of the conversion of potential energy into radiation. For a $1 M_{\odot}$ white dwarf of radius $5 \times 10^{8} \mathrm{~cm}$, the corresponding mass transfer rate is $\dot{m}=3.7 \times 10^{16} \mathrm{~g} \mathrm{~s}^{-1}$, substantially smaller than that derived by a conventional analysis $\left(\dot{m}=3 \times 10^{17} \mathrm{~g} \mathrm{~s}^{-1}\right.$ : Smak 1982 ; Patterson 1984). The reason for this discrepancy is that our inferred energy distribution requires a very small bolometric correction (the bulk of the radiation falls in the optical and ultraviolet). On the other hand, theoretical disks with boundary layers radiate a large fraction of their luminosity in the extreme-ultraviolet (see Fig. 4). If it turns out that conventional analyses systematically overestimate the mass transfer rate, then this would solve the conundrum posed by Ferland et al. (1982) regarding the X-ray luminosities of classical novae.

\section{c) Model Calculations for the Nebula Surrounding $D Q \mathrm{Her}$}

We have performed photoionization calculations with the program described by Ferland and Truran (1981; see the recent description in Ferland and Mushotzky 1982). A major difference between these calculations and previous studies is the improved treatment of dielectronic recombination at low temperatures, as described above and by Nussbaumer and Storey (1983). The fitting formulae given by Nussbaumer and Storey are not valid for temperatures lower than $1000 \mathrm{~K}$, but for the majority of the ions of interest radiative recombination dominates at such low temperatures. The entire atomic data base has been revised in light of the compendium by Mendoza (1983). Radiative recombination coefficients for $10^{4} \mathrm{~K}$ are now taken from Gould (1978), with the temperature scaling laws of Aldrovandi and Péquignot (1971). An exception is iron, which is taken from Woods, Shull, and Sarazin (1982). All groundstate and inner-shell photoionization cross sections are now from the calculations of Reilman and Manson (1979). In the present calculations diffuse radiation fields of the heavy elements, important because of their enriched abundances, are treated in the outward-only approximation.

It is important to keep in mind that, because of the extremely low temperatures, a comparison between photoionization calculations and these cool nebulae tests our understanding of the relevant atomic physics (see Osterbrock 1982; Flower, Penn, and Seaton 1982; for a discussion in a different context). This is especially true of the present study: the physical conditions encountered here are unprecedented and we have no guarantee that existing treatments of recombination, charge exchange, and inelastic electron scattering are reliable. We cite the example of charge transfer, which is particularly important both as a neutralization process for the heavy elements and as an ionization process for hydrogen. The present rate coefficients are taken from the quantal calculations of Butler, Heil, and Dalgarno (1980), who give predictions for the temperature range $5000<T_{e}<20,000 \mathrm{~K}$. We have extrapolated these rates to $500 \mathrm{~K}$. This extrapolation could easily be off by factors of 0.5 dex. Similarly, because of the low temperatures, essentially all inelastic scattering processes occur to threshold, where atomic theory is at its worst.

We have used the ionizing continua in Figures 4 and 5, and assumed a constant-density nebula with thickness $\delta r=1.4 \times 10^{16} \mathrm{~cm}$ at a distance $3.7 \times 10^{16} \mathrm{~cm}$ from the accreting binary. A volume filling factor of $\frac{2}{3}$ was assumed. The mass transfer rate was varied between $10^{16} \mathrm{~g} \mathrm{~s}^{-1}$ and $10^{18.5} \mathrm{~g}$ $\mathrm{s}^{-1}$ to fully encompass the nova phenomenon; we also use the more realistic ionizing continuum shown as a thin line in Figure 5. The best estimate of the gas density is $N=10^{2} \mathrm{~cm}^{-3}$ (see above), and we vary $N$ between $10^{1} \mathrm{~cm}^{-3}$ and $10^{4} \mathrm{~cm}^{-3}$. The chemical composition is an important ingredient. Ferland and Truran (1981) used a mixture similar to that measured by 
Ferland and Shields (1978) for the very fast nova V1500 Cygni (viz., C, N, and $\mathrm{O}$ enhancements of 20,100 , and 20 relative to the solar mixture of Lambert 1978 and Lambert and Luck 1978). It now seems clear that many novae, and DQ Her in particular, have much more extreme enhancements (Williams $1982 b$ ). With the exception of oxygen (to be discussed below), we employ the mixture measured by Martin and Ferland (1983) using archival spectrographic data from early phases of DQ Her's outburst; e.g., helium, carbon, nitrogen, and oxygen are enriched by factors of $3,100,300$, and 30 , respectively.

\section{d) Results}

Given the ionizing continua shown in Figures 4 and 5, we find that two ingredients, a low density and an enhanced oxygen abundance, are needed to achieve a low electron temperature. It has long been known that infrared fine-structure lines, particularly the B I and $\mathrm{C}$ I sequences of carbon, nitrogen, and oxygen, are important coolants for low-density nebulae (Burbidge, Gould, and Pottasch 1963). For the ionization conditions and chemical composition appropriate for the nebula surrounding DQ Her, the $88 \mu \mathrm{m}$ and $52 \mu \mathrm{m}$ lines of [O $\mathrm{III}$ ] are extremely efficient coolants and can easily cool the gas to 500 $\mathrm{K}$.

Table 3 shows the effects of the [O III] lines on various models. These calculations assume solar abundances for all elements other than oxygen, a density of $10^{2} \mathrm{~cm}^{-3}$, the ionizing continuum shown in Figure 5, and the nebular dimensions given above. The oxygen abundance was varied between onethird and 100 times the solar $\mathrm{O} / \mathrm{H}$ ratio. The table shows the average temperature and the fraction of the total cooling carried by the [O III] fine-structure lines. For low oxygen abundances the result is a fairly classical nebula, with optical forbidden lines carrying the bulk of the cooling. As the oxygen abundance increases, the temperature falls and the finestructure lines carry an ever increasing share of the total cooling. For abundances greater than 20 times solar, the finestructure lines carry essentially all the cooling and the predicted temperatures match the observed low temperatures found. It is important to keep in mind that the key parameter in Table 3 is not the total oxygen abundance, but rather the abundance of the $\mathrm{O}^{+2}$ ion. Little $\mathrm{O}^{+3}$ is present, and although a fair fraction of the oxygen is in the form of $\mathrm{O}^{+}$, this ion has no excited level accessible at low temperatures. Charge exchange with hydrogen helps to determine the ionization fraction of $\mathrm{O}^{+2}$ (see Butler, Heil, and Dalgarno 1980), and, as was stressed above, charge exchange rate coefficients at low temperatures are poorly known. The equilibrium temperature varies roughly linearly with the $\mathrm{O}^{+2}$ abundance, so one could match the observed temperature by varying either the total oxygen abun-

TABLE 3

Thermal EQUILIBRIA

\begin{tabular}{ccc}
\hline \hline$[\mathrm{O} / \mathrm{H}]^{\mathrm{a}}$ & $\left\langle T_{e}\right\rangle(\mathrm{K})$ & $L(52,88 \mu \mathrm{m}) /$ Total $^{\mathrm{b}}$ \\
\hline $0.33 \ldots \ldots \ldots$ & 8650 & 0.08 \\
$1.00 \ldots \ldots \ldots$ & 6570 & 0.28 \\
$3.00 \ldots \ldots \ldots$ & 2750 & 0.52 \\
$10.0 \ldots \ldots \ldots$ & 1150 & 0.73 \\
$30.0 \ldots \ldots \ldots$ & 342 & 0.75 \\
$100 \ldots \ldots \ldots$ & 180 & 0.79 \\
\hline
\end{tabular}

a Oxygen enhancement relative to a solar abundance.

${ }^{b}$ Fraction of total cooling carried by $[\mathrm{O}$ III $]$ finestructure lines. dance or the $\mathrm{O}^{+2} \rightarrow \mathrm{O}^{+}$charge exchange rate coefficient. It is difficult to say which has the greater uncertainty. We have chosen to set the oxygen abundance to a value 30 times greater than solar, with the understanding that this abundance absorbs the error associated with the uncertainty in the charge exchange rate coefficient. For comparison, Martin and Ferland (1983) deduced an oxygen abundance some 0.5 dex larger from an analysis of oxygen recombination lines observed during an earlier portion of the nebular phase. With this one exception, we will employ the Martin and Ferland mixture in the remainder of our analysis.

Interestingly enough, it turns out that enhancements of other elements (particularly helium and carbon) raise the temperature of the gas rather than lower it. This is because these elements add additional opacity to the mixture (and hence increase the photoelectric heating rate) without contributing to the cooling efficiency.

The $[\mathrm{O} \mathrm{III}]$ fine-structure lines are efficient coolants provided that the gas density is below the critical density for the $J=1,2$ sublevels of the $\mathrm{O}^{+2}{ }^{3} P$ ground state $\left(N=670 \mathrm{~cm}^{-3}\right.$ and $4900 \mathrm{~cm}^{-3}$, respectively; Osterbrock 1974). To see this, consider the results shown in Figure 6. An ionization parameter $U$ is defined as the ratio of photon density to electron density: $U=Q(\mathrm{H}) / 4 \pi r^{2} N c$ where $Q(\mathrm{H})$ is the number of ionizing photons emitted by the central object per second (Osterbrock 1974), $r$ is the source-cloud separation, and $N$ and $c$ are the gas density and speed of light, respectively. This parameter is useful because of homology relations describing the ionization and thermal structure of models with different electron densities but similar values of $U$ (see Tarter, Tucker, and

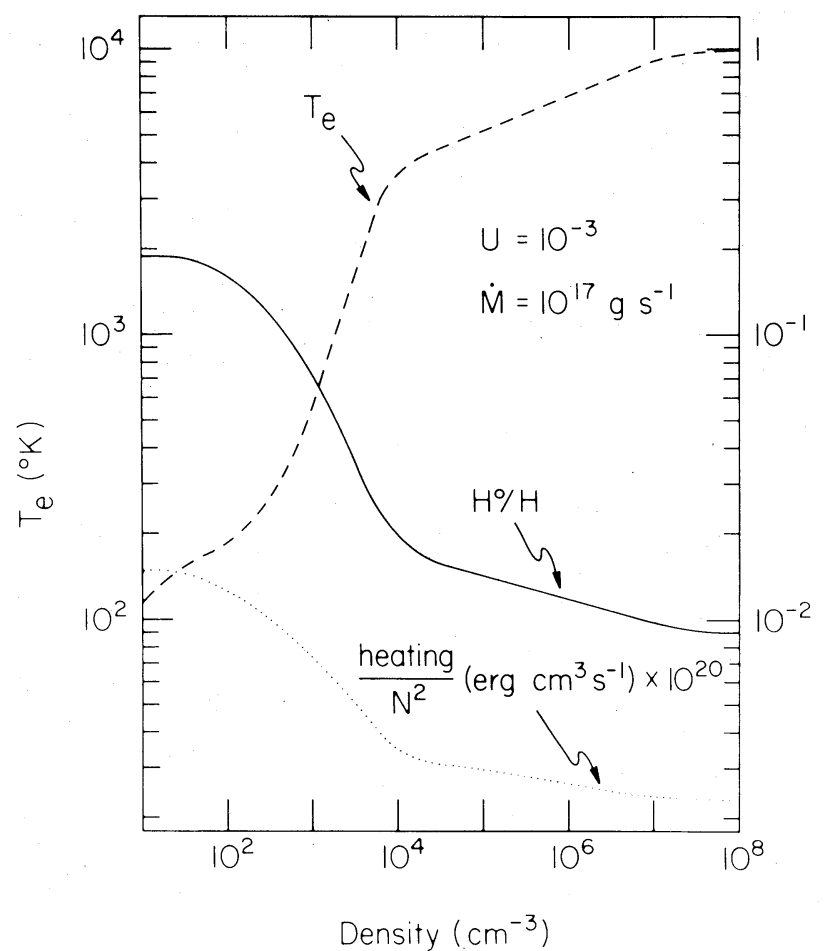

Fig. 6.-Physical conditions. This figure shows the dependence of the physical conditions on the gas density. These calculations are for an optically thin gas with the enriched chemical composition and $[\mathrm{O} / \mathrm{H}]=100$. The ionization parameter is held fixed at $U=10^{-3}$ as the density is varied. The temperature falls dramatically at $N<10^{3} \mathrm{~cm}^{-3}$ because the infrared fine-structure lines become efficient coolants. The hydrogen neutral fraction and the heating rate (both plotted) increase as $T_{e}$ falls because the radiative recombination coefficients increase roughly as $T^{-0.8}$. 
Salpeter 1969; Davidson and Netzer 1979). If the cooling efficiency of the gas were to remain constant, models with similar $U$ but different densities would be identical.

To illustrate the effects the [O III] fine-structure lines have, Figure 6 shows the results for a grid of models in which the ionization parameter was held fixed at $U=10^{-3}$ and the density varied between the extremes encountered during the nebular phase of the nova outburst $\left(10^{1} \mathrm{~cm}^{-3}<N<10^{8}\right.$ $\mathrm{cm}^{-3}$ ). The calculations are for our adopted mixture and an ionizing continuum corresponding to a mass transfer rate of $10^{17} \mathrm{~g} \mathrm{~s}^{-1}$. At high densities only ultraviolet resonance lines are efficient coolants and the gas equilibriates at $T \approx 10^{4} \mathrm{~K}$. The temperature falls to $\sim 6000 \mathrm{~K}$ when the optical forbidden lines (mainly [O III] $\lambda \lambda 5007,4959$ ) begin to cool the gas $\left(N<10^{6} \mathrm{~cm}^{-3}\right)$. Finally, the temperature falls below $\sim 10^{3} \mathrm{~K}$ when the infrared fine-structure lines are no longer collisionally deactivated $\left(N<10^{3} \mathrm{~cm}^{-3}\right)$. This low temperature is the result of both the high abundances and the low densities, which together produce very efficient fine-structure cooling at low temperatures.

Figure 6 also shows the heating rate coefficient, the hydrogen neutral fraction, and the doubly to singly ionized carbon ratio. Both increase as the density falls for a fixed $U$, because the radiative recombination coefficient, $\alpha\left(T_{e}\right)$, increases roughly as $T^{-0.8}$. It is easily shown that, in steady state, the neutral fraction is $N^{0} / N^{+}=\alpha\left(T_{e}\right) / U c \sigma$ when the gas is mainly ionized. Similarly, the heating rate in steady state is proportional to the recombination rate coefficient. The fact that the gas still manages to attain such low temperatures despite this larger heating rate coefficient is a testimonial to the efficiency of the fine-structure cooling.

The results of a large number of calculations are summarized in Table 4 for both the empirical and theoretical continua described in $\S$ IV. The table gives the mean temperature and the $\mathrm{C}^{+2} / \mathrm{C}^{+}$ratio for the models. For a given continuum, the level of ionization increases as the density is lowered. This would also cause the temperature to increase were the efficiency of the fine structure line cooling not also increasing. The net result is that the temperature falls with decreasing density (and increasing cooling efficiency) until the level of ionization of the gas is so large that $\mathrm{C}, \mathrm{N}$, and $\mathrm{O}$ have reached ionization stages which have no prominent fine-structure lines (e.g., the

TABLE 4

Physical Conditions

\begin{tabular}{ccccc}
\hline \hline & \multicolumn{4}{c}{$N_{\mathrm{H}}\left(\mathrm{cm}^{-3}\right)$} \\
\cline { 2 - 5 } Continuum & $10^{1}$ & $10^{2}$ & $10^{3}$ & $10^{4}$ \\
\hline Observed $\ldots \ldots \ldots \ldots$ & $238 \mathrm{~K}^{\mathrm{a}}$ & $613 \mathrm{~K}$ & $3040 \mathrm{~K}$ & $3390 \mathrm{~K}$ \\
& $6.3^{\mathrm{b}}$ & 1.1 & 0.05 & 0.003 \\
$16.0^{\mathrm{c}} \ldots \ldots \ldots \ldots \ldots \ldots$ & $333 \mathrm{~K}$ & $1440 \mathrm{~K}$ & $3440 \mathrm{~K}$ & $4110 \mathrm{~K}$ \\
& 3.52 & 0.60 & 0.03 & 0.004 \\
$16.5 \ldots \ldots \ldots \ldots \ldots \ldots$ & $460 \mathrm{~K}$ & $736 \mathrm{~K}$ & $3650 \mathrm{~K}$ & $3610 \mathrm{~K}$ \\
& 9.94 & 1.47 & 0.078 & 0.005 \\
$17.0 \ldots \ldots \ldots \ldots \ldots \ldots$ & $11400 \mathrm{~K}$ & $497 \mathrm{~K}$ & $3750 \mathrm{~K}$ & $3870 \mathrm{~K}$ \\
& 52.7 & 3.41 & 0.234 & 0.013 \\
$17.5 \ldots \ldots \ldots \ldots \ldots \ldots$ & $16400 \mathrm{~K}$ & $683 \mathrm{~K}$ & $3660 \mathrm{~K}$ & $3950 \mathrm{~K}$ \\
& 212 & 12.5 & 0.935 & 0.035 \\
$18.0 \ldots \ldots \ldots \ldots \ldots \ldots$ & $22000 \mathrm{~K}$ & $12900 \mathrm{~K}$ & $3020 \mathrm{~K}$ & $4090 \mathrm{~K}$ \\
& 728 & 68 & 3.93 & 0.099 \\
$18.5 \ldots \ldots \ldots \ldots \ldots \ldots$ & $33900 \mathrm{~K}$ & $18000 \mathrm{~K}$ & $5010 \mathrm{~K}$ & $4650 \mathrm{~K}$ \\
& 3180 & 251 & 12.9 & 0.318 \\
\hline
\end{tabular}

a Mean temperature (observed is $\sim 500 \mathrm{~K}$ ).

${ }^{b}$ Mean $\mathrm{C}^{++} / \mathrm{C}^{+}$(observed is $\sim 1.3$ ).

${ }^{c}$ Log of mass transfer rate $\left(\mathrm{g} \mathrm{s}^{-1}\right)$. lithium sequence). When this low density is reached, the cooling is achieved with optical and ultraviolet lines, and the temperature rises to $10^{4} \mathrm{~K}$.

These calculations show that the gas can attain low temperatures for a wide variety of radiation fields. The low temperatures seen in shells around DQ Her and some other novae are a general characteristic of metal-rich nebulae. Little can be deduced from the electron temperature alone about either the far-ultraviolet continuum or the chemical composition. It is then no surprise that such dramatically different systems as DQ Her and CP Pup (Williams 1982a) have nearly identical nebulae.

Table 5 lists detailed predictions of the model with the continuum shown in Figure 5 and $N=10^{2} \mathrm{~cm}^{-3}$. No attempt has been made to "fine tune" the model. The differences between the observed and predicted spectra are within the very considerable observational and theoretical uncertainties (judged to be $\sim 0.5 \mathrm{dex}$ ). All ultraviolet and optical lines are formed by recombination, with the exception of the $[\mathrm{O}$ II $] \lambda 3727$ doublet, which is predominantly produced by photoionization into excited states (Ferland and Truran 1981). The strongest infrared fine-structure lines are also listed. These predictions offer an observational test of our model.

\section{CONCLUSIONS}

In this paper we have shown that ultraviolet and optical observations of the nebula surrounding DQ Her confirm Williams et al.'s (1978) measurement of an unprecedentedly low temperature. We further show that the gas is fairly highly ionized and that its cooling and recombination times are shorter than the expansion time of the shell. The shell must have a source of heat and ionization. The physical conditions deduced here rule out both Williams et al.'s (1978) suggestion that the ionization is frozen in and Ferland and Truran's (1981) model invoking an X-ray source to power the shell.

New ultraviolet observations of the underlying binary are combined with published X-ray, optical, and infrared measurements and inferred extreme-ultraviolet flux to deduce the complete energy distribution of radiation from the accreting binary. This emergent continuum bears no resemblance to theoretical predictions for a steady-state accretion disk with boundary layer.

The direct measurement of the bolometric luminosity of the central object allows us to measure the accretion rate directly. The result is a factor of 10 lower than that derived by conven-

TABLE 5

Emission-Line Predictions

\begin{tabular}{|c|c|c|}
\hline Ion & $\lambda$ & Intensity \\
\hline $\mathrm{H}_{\mathrm{I}} \ldots$ & $4861 \AA$ & 100 \\
\hline $\mathrm{H}$ I $\ldots \ldots$ & $6563 \AA$ & 250 \\
\hline He I $\ldots$. & $5876 \AA$ & 45 \\
\hline He il $\ldots \ldots \ldots \ldots \ldots$ & $4686 \AA$ & 22 \\
\hline $\mathrm{C}_{\mathrm{I}} \ldots \ldots \ldots \ldots \ldots \ldots$ & $1656 \AA$ & 15 \\
\hline $\mathrm{C}$ II $\ldots \ldots \ldots \ldots \ldots$ & $1335 \AA$ & 82 \\
\hline $\mathrm{C}$ II $\ldots \ldots \ldots \ldots \ldots \ldots$ & $4267 \AA$ & 16 \\
\hline $\mathrm{C}$ III $] \ldots \ldots \ldots \ldots \ldots$ & $1909 \AA$ & 21 \\
\hline $\mathrm{N}$ II $\ldots \ldots \ldots \ldots \ldots$ & $5005 \AA$ & 24 \\
\hline$[\mathrm{N} \mathrm{II}]$. & $6548,6584 \AA$ & 12 \\
\hline$\left[\begin{array}{ll}\mathrm{O}_{1}\end{array}\right] \ldots \ldots \ldots \ldots \ldots$. & $6300 \AA$ & 0 \\
\hline$\left[\begin{array}{lll}\mathrm{O} & \mathrm{II}\end{array}\right]$. & $3727 \AA$ & 24 \\
\hline$[\mathrm{O}$ III $] \ldots \ldots \ldots \ldots \ldots$ & $88,52 \mu \mathrm{m}$ & 1920 \\
\hline$[\mathrm{O}$ IV $] \ldots \ldots \ldots \ldots \ldots$ & $26 \mu \mathrm{m}$ & 92 \\
\hline$[\mathrm{Si} \mathrm{II}] \ldots \ldots \ldots \ldots \ldots$ & $35 \mu \mathrm{m}$ & 28 \\
\hline$[\mathrm{S}$ III $] \ldots \ldots \ldots \ldots \ldots$ & $34 \mu \mathrm{m}$ & 16 \\
\hline
\end{tabular}


tional analysis. If this result is a general property of novae, then the apparent X-ray deficiency of many systems, as well as the fact that many objects appear to be accreting at too large a rate to undergo a nova outburst (Fujimoto 1982; Prialnik et al. 1982; MacDonald 1983), would be understood. Similar analyses on other old novae with appreciable shells should be performed to derive empirical bolometric luminosities and mass transfer rates.

Photoionization calculations are presented to show that a cool nebula can be produced by photoionization with either the observed continuum or that from a model accretion disk with boundary layer. In particular, this suggests that both the very high metallicity deduced by Williams $(1982 b)$ and the present treatment of the relevant atomic physics at low temperatures are essentially correct. The low temperature is the result of a combination of very high metal abundances and low density. The infrared fine-structure lines, particularly the 52,88 $\mu \mathrm{m}$ lines of $[\mathrm{O} \mathrm{III}]$, are very efficient coolants, and the nebula easily reaches equilibrium at low temperatures. Unfortunately, this result is fairly independent of the radiation field used to ionize the gas, and thus little can be inferred about the unobservable far-ultraviolet continuum emitted by the underlying binary. This may explain why shells surrounding many novae are now known to have low temperatures. Indeed, McLaughlin (1942) identifies the disappearance of the [O III] $\lambda \lambda 5007,4959$ lines as a common feature of the late nebular phase of many novae. Williams (1982a) finds that, although many nova shells are cold, some are not. This raises the interesting question of why such a dichotomy should exist.

Our calculations suggest that high temperatures should prevail in shells which are too dense for fine-structure cooling to be important, or have a very large value of the ionization parameter (so that little $\mathrm{O}^{++}$is present), or are far less metal- rich than DQ Her. We reject the possibility that the very rapidly expanding shells surrounding very old novae have not yet reached a low enough density for fine-structure cooling to be important. The last possibility (lower metallicity in some novae) is intriguing because, in the conventional picture of the nova outburst (Truran 1982), fast novae should be relatively metal-rich. However, both the relatively slow nova DQ Her and the luminous, very fast nova CP Pup have cold shells, so no trend is apparent.

The need for infrared observations is obvious. As can be seen from Table 4, these cold nova shells should be powerful radiators of infrared emission lines. Together, the [O III] finestructure lines should have an observed flux of $2.7 \times 10^{-12}$ ergs $\mathrm{cm}^{-2} \mathrm{~s}^{-1}$. Far-infrared observations would provide a valuable measure of the true metal abundances of these DQ Her-like shells.

Cold novae shells offer two avenues for future research. First, because of the low temperature, virtually all atomic processes occur right at threshold, where theory is usually at its worst. If one knew a priori the chemical composition of the shell (say, from an extensive analysis of observations of the early nebular phase when the physical conditions are more classical), then several charge-exchange and recombination rate coefficients could be measured directly. Similarly, these nebulae present an opportunity to measure the far-UV continua from cataclysmic variables directly. The fact that the continuum determined here is at such great odds with theory is intriguing.

G. J. F. thanks Joe Patterson for several discussions and the NSF for support through grant AST 83-05094. This research has been supported in part by NASA through grant NSG5379 .

\section{REFERENCES}

Aldrovandi, S. M. V., and Péquignot, D. 1973, Astr. Ap., 25, 137

Bath, G. T., Pringle, J. E., and Whelan, J. A. J. 1980, M.N.R.A.S., 190, 185

Becker, R. H., and Marshall, R. E. 1981, Ap. J. (Letters), 244, L93.

Bianchini, A., and Sabbadin, F. 1982, Proc. Third European IUE Conference.

Burbidge, G. R. Gould, R. J, and Pottasch, S. R. 1963, Ap. J., 138, 945.

Butler, S., Heil, T., and Dalgarno, A. 1980, Ap. J., 241, 442.

Cohen, J. G., and Rosenthal, A. J. 1983, Ap. J., 268, 689.

Córdova, F. A., Mason, K. O., and Nelson, J. E. 1981, Ap. J., 245, 609

Davidson, K., and Netzer, H. 1979, Rev. Mod. Phys., 51, 715.

de Vaucouleurs, G., de Vaucouleurs, A., and Corwin, H. G. 1976, Second

Reference Catalogue of Bright Galaxies (Austin: University of Texas), p. 32

Fabbiano, G., et al. 1981, Ap. J., 243,911.

Fabian, A. C., Pringle, J. E., and Rees, M. J. 1976, M.N.R.A.S., 175, 43.

Ferland, G. J. 1980a, Observatory, 100, 166

. 1980b, Pub. A.S.P., 92, 596.

Ferland, G. J., Langer, S. H., MacDonald, J., Pepper, G. H., Shaviv, G., and

Truran, J. W. 1982, Ap. J. (Letters), 262, L53.

Ferland, G. J., and Mushotzky, R. F. 1982, Ap. J., 262, 564.

Ferland, G. J., and Shields, G. A. 1978, Ap. J., 226, 172.

Ferland, G. J., and Truran, J. W. 1981, Ap. J., 244, 1032

Flower, D. R., Penn, C. J., and Seaton, M. J. 1982, M.N.R.A.S., 201, 39p.

Fujimoto, M. 1982, Ap. J., 257, 752.

Gallagher, J S., Hege, E. K, Kopriva, D. A., Williams, R. E., and Butcher, H. R. 1980, Ap. J., 237, 55 .

Gallagher, J.S., and Starrfield, S. 1978, Ann. Rev. Astr. Ap., 16, 171.

Gould, R. J. 1978, Ap. J., 219, 250.

Hartmann, L., and Raymond, J. 1981, in The Universe at $U V$ Wavelengths, ed. R. O. Chapman (NASA Conf. Pub. 2171), p. 495.

Hassall, B. J. M., Pringle, J. E., Schwarzenberg-Czerny, A., Wade, R. A. Whelan, J. A. J., and Hill, P. W. 1983, M.N.R.A.S., 203, 865

Hutchings, J. B., Cowley, A. P., and Crampton, D. 1979, Ap. J., 232, 500.

Kiplinger, A. L. 1979, Ap. J., 234, 997.
Kurucz, R. L., and Peytremann, E. 1975, SAO Spec. Rept., No. 362.

Kylafis, N., and Lamb, D. 1979, Ap. J. (Letters), 228, L105.

Lambert, D. L. 1978, M.N.R.A.S., 182, 249

Lambert, D. L., and Luck, R. E. 1978, M.N.R.A.S., 183, 79

MacDonald, J. 1983, Ap. J., 267, 732.

Martin, P. G., and Ferland, G. J. 1983, in preparation.

McLaughlin, D. B. 1942, Ap. J., 95, 428.

Mendoza, C. 1983, in IAU Symposium 103, Planetary Nebulae, ed. D. R. Flower (Dordrecht: Reidel).

Moore, C. E. 1970, Selected Tables of Atomic Spectra (NSRDS-NBS 3).

Nussbaumer, H., and Storey, P. J. 1983, Astr. Ap., in press.

Osterbrock, D. E. 1974, Astrophysics of Gaseous Nebulae (San Francisco: Freeman).

. 1982, in IAU Symposium 103, Planetary Nebulae, ed. D. R. Flower (Dordrecht: Reidel)

Payne-Gaposchkin, C., and Whipple, F. 1939, Harvard Circ., No. 433.

Patterson, J. 1984, Ap. J. Suppl., 54, 443.

Patterson, J., Robinson, E. L., and Nather, R. E. 1978, Ap. J., 224, 570

Pottasch, S. 1959, Ann. d'Ap., 22, 394.

Prialnik, D., Livio, M., Shaviv, G., and Kovetz, A. 1982, Ap. J., 257, 312

Pringle, J. E. 1977, M.N.R.A.S., 178, 195.

1981, Ann. Rev. Astr. Ap.,19,137.

Pringle, J. E., and Savonije, G. 1979, M.N.R.A.S., 187, 777

Reilman, R. F., and Manson, S. T. 1979, Ap. J. Suppl., 40, 111.

Schneider, D. P., and Greenstein, J. L. 1979, A p. J., 233, 935.

Seaton, M. J. 1978, in Planetary Nebulae, Observations and Theory, ed. Y. Terzian, p. 131.

Tmak, J. 1979, M.N.R.A.S., 187, 73p.
Acta Astr., 32, 213 .

Stickland, D. J., Penn, C. J., Seaton, M. J., Snijders, M. A. J., and Storey, P. J.

1981, M.N.R.A.S., 197, 107

Storey, P. J. 1981, M.N.R.A.S., 195, 27p. 
Tarter, C. B., Tucker, W. H., and Salpeter, E. E. 1969, Ap. J., 156, 943.

Truran, J. 1982, in Essays in Nuclear Astrophysics, ed. C. Barnes (Cambridge:

Cambridge University Press), in press.

Tylenda, R. 1977, Acta Astr., 27, 235

Williams, R. E. 1980, Ap.J., 235, 939
Williams, R. E. $1982 a, A p . J, 261,170$

1982b, Ap.J.(Letters), 261, L71.

Williams, R. E., Woolf, N. J., Hege, E. K., Moore, R. L., and Kopriva, D. A. 1978, Ap. J., 224, 171

Woods, D. J., Shull, J. M., and Sarazin, C. L. 1982, Ap. J., 257, 918

Note added in proof-After this work was completed, we found that Itoh (Pub. Astr. Soc. Japan, 33, 743-747) had reached essentially the same conclusions as we concerning the origin of the cold nova shells.

GARY J. FERLAND: Department of Physics and Astronomy, University of Kentucky, Lexington, KY 40506

P. M. GondhaleKar: Rutherford Appleton Laboratory, Chilton, Didcot, Oxfordshire OX11 0QX, England, UK

David L. Lambert, Gregory A. Shields, and Mark Slovak: Department of Astronomy, University of Texas, Austin, TX 78712

JAMES W. TRURAN : Astronomy Department, University of Illinois, 341 Astron. Bldg., Urbana, IL 61801

Robert E. Williams: Steward Observatory, University of Arizona, Tucson, AZ 85721 